\title{
Three-halves harmonic emission from femtosecond laser produced plasmas
}

L. Veisz, W. Theobald, T. Feurer, H. Schillinger, P. Gibbon, and R. Sauerbrey

Institut für Optik und Quantenelektronik, Friedrich-Schiller-Universität, D-07743 Jena, Germany

M. S. Jovanović

Institute for Laser Engineering, University of Osaka, Suita, Osaka 565-0871, Japan

(Received 25 February 2002; accepted 21 May 2002)

\begin{abstract}
Measurements of three-halves harmonic radiation $\left(3 \omega_{0} / 2\right)$ produced by femtosecond, Ti:sapphire laser pulses $\left(\leqslant 2 \times 10^{17} \mathrm{~W} / \mathrm{cm}^{2}\right)$ in long density scale length plasmas generated from solid aluminum targets are presented. The $3 \omega_{0} / 2$ emission yield shows excellent agreement with theories of the two-plasmon decay instability in the predominantly linear regime. (C) 2002 American Institute of Physics. [DOI: 10.1063/1.1493794]
\end{abstract}

High intensity femtosecond laser produced plasmas form the basis of important research topics such as x-ray lasers, laser driven particle accelerators schemes, laser initiated nuclear reactions, and relativistic effects in laser-plasmas. ${ }^{1}$ The interaction of intense ultrashort laser pulses with solids leads to the generation of solid density plasmas ${ }^{2}$ with electron temperatures of several $\mathrm{keV}$. Such plasmas are sources of strong x-ray and particle emission with energies of up to several $10 \mathrm{MeV}$. In addition to resonance absorption ${ }^{3,4}$ parametric plasma instabilities contribute to the generation of suprathermal electrons and also lead to the generation of large amplitude plasma waves which couple to the incident electromagnetic wave and result in new, frequency-shifted electromagnetic emission. An example of such a process is the generation of light with a frequency of $3 / 2$ times the fundamental frequency. In the interaction of intense Ti:sapphire femtosecond laser pulses with solids, this manifests itself as a strong green emission, ${ }^{5}$ and is a signature of parametric instabilities operating close to quarter critical density $\left(1 / 4 n_{c}\right)$.

Plasma instabilities are an important topic for laser fusion and considerable attention has been directed to the question of energy coupling into the plasma and its potential for generating high-energy electrons which preheat the fuel. Plasma instabilities have been extensively studied for many years in laser-plasma experiments with pulse durations between about 100 ps and 20 ns. In particular, stimulated Raman scattering (SRS), where the incident photon decays into a scattered photon and a plasmon, and the two-plasmon decay instability (TPD) which is the parametric decay of an incident laser photon into two plasmons, are relevant processes. ${ }^{6-10}$

In long pulse laser-plasma interaction, the dominant production process for 3/2-harmonic radiation was found to be TPD, which occurs resonantly at $1 / 4 n_{c} .{ }^{10}$ With nanosecond and sub-nanosecond pulses, the instability reaches a nonlinear saturation caused by the decay of an electron plasma wave into an ion-acoustic wave and a scattered electron plasma wave (Langmuir wave decay instability-LDI). ${ }^{11}$ Time-resolved Thompson scattering measurements in $\mathrm{CO}_{2}$ laser-plasma interaction experiments at $3 \times 10^{13} \mathrm{~W} / \mathrm{cm}^{2}$ (with $2 \mathrm{~ns}$ pulse length) revealed that for times up to $25 \mathrm{ps}$, TPD growth is within a linear regime; whereas after $50 \mathrm{ps,}$ saturation occurs. ${ }^{10}$ However, the saturation timescale depends on the laser and plasma conditions and therefore will be different in other experiments. Typical TPD saturation levels of the enhanced electron plasma fluctuations for nanosecond laser-pulse experiments are a factor of $10^{3}$ to $10^{4}$ (7 to $9 e$-foldings) above thermal noise levels. ${ }^{12}$

In the femtosecond regime, however, the product of growth rate and pulse duration is much smaller, so that one would expect analogous measurements to fall within the linear regime. In addition, almost no hydrodynamic motion occurs during the interaction time. Although several groups have reported SRS-backscatter measurements in underdense plasmas $\left(n_{e}<0.01 n_{c}\right),{ }^{13}$ to the best of our knowledge no investigations of $3 \omega_{0} / 2$ generation by femtosecond lasers have been performed to date.

This article reports first experimental studies of high intensity fs-laser generated $3 \omega_{0} / 2$ radiation in inhomogeneous plasmas. We show that measurements of the 3/2-harmonic yield as a function of the incident pulse duration are in excellent agreement with linear theories. A 2-TW Ti:sapphire laser beam (center wavelength $\lambda_{0}=790 \mathrm{~nm}$, Fig. 1) delivering 100-fs, 200-mJ laser pulses with a repetition rate of 10 $\mathrm{Hz}$ was used for the experiment. The beam propagated from the last compressor grating a distance of $4 \mathrm{~m}$ in air before entering a target chamber filled with air with a low ambient pressure of 5 mbar. This was chosen to reduce the debris from the target which tends to degrade the focusing optics especially when operating at $10 \mathrm{~Hz}$. Nonlinear effects in air and in the $10 \mathrm{~mm}$ thick fused silica entrance window caused a pulse prolongation which resulted in an effective pulse duration of about $135 \mathrm{fs}$ on target. The p-polarized laser was focused to a spot size of $(20 \pm 5) \mu \mathrm{m}$ with an off-axis parabolic mirror onto a polished aluminum target with $45^{\circ}$ angle of incidence and with an aperture angle of $23^{\circ}$ ( $f / 2.5$ focusing). Nonlinear phase front distortions did not affect the measured spot size. For each laser shot a fresh surface was provided by shifting the target. Including reflection losses at the 


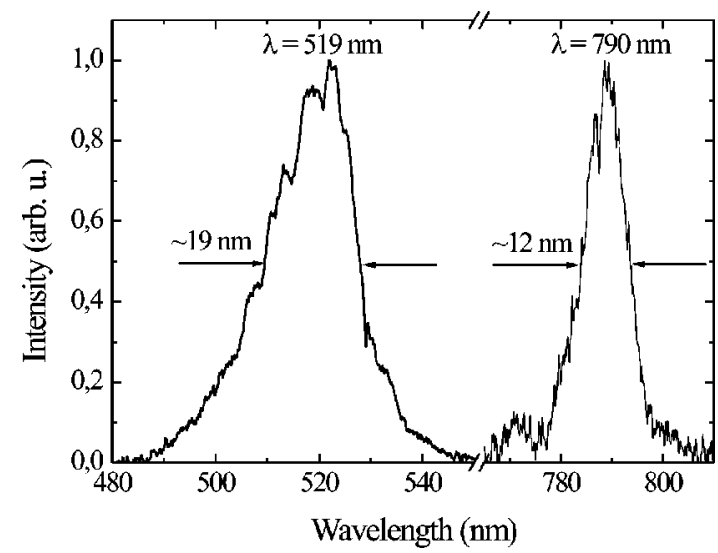

FIG. 1. Measured $3 \omega_{0} / 2$ spectrum averaged over 15 laser shots and the spectrum of the fundamental.

window and at a pellicle shield in front of the parabolic mirror we obtained an intensity of $(2.3 \pm 1.1) \times 10^{17} \mathrm{~W} / \mathrm{cm}^{2}$ in vacuum, which is not reduced by ionization defocusing in air at a pressure of 5 mbar in our chamber. ${ }^{14}$ The plasma is produced by a prepulse appearing $12.5 \mathrm{~ns}$ before the main pulse with an intensity of about $4 \times 10^{14} \mathrm{~W} / \mathrm{cm}^{2}$. This prepulse was generated by allowing some leakage of the previous oscillator pulse through the Pockels cell of the regenerative amplifier. In addition, we measured a prepulse at 4 ps before the intense main pulse with about the same intensity as the $12.5 \mathrm{~ns}$ prepulse by means of a third order autocorrelation.

The generated $3 \omega_{0} / 2$ radiation was collimated in $45^{\circ}$ observation angle with a second off-axis parabolic mirror and sent through a BK7 window to the diagnostics. The energy of the $3 \omega_{0} / 2$ signal was measured carefully with a calibrated photodiode provided with an appropriate filtering and yielded approximately $100 \mathrm{~nJ}$ per pulse (average of $\sim 50$ pulses) into a solid angle of $\Delta \Omega \approx 2 \times 10^{-3}$ sr. Strong shot to shot energy fluctuations were observed. The conversion efficiency of the incident energy into 3/2-harmonic energy which was measured in specular direction is $5 \times 10^{-7}$. We measured also the second-harmonic emission from the plasma with a pulse energy of about $50 \mathrm{~nJ}$.

Figure 1 shows an averaged spectrum which was obtained by integrating over 15 shots and the incident laser spectrum. The spectral details varied from shot to shot but the spectral width and the center wavelength were essentially invariant. The spectrum with a full width at half maximum of $(19 \pm 1) \mathrm{nm}$ is centered at a wavelength of $(519 \pm 1) \mathrm{nm}$ with about $8 \mathrm{~nm}$ blue shift and confirms that the observed radiation is due to $3 / 2$-harmonic emission. In contrast to nanosecond laser experiments where typically well separated red and a blue shifted wings are observed, ${ }^{8,9}$ we measure only a broad spectral distribution. For nanosecond laser experiments the 3/2-harmonic spectrum is explained by simulations including nonlinear TPD saturation and LDI. ${ }^{11}$ Obviously, the 3/2-harmonic spectrum shown in Fig. 1 indicates that with femtosecond lasers a completely different coupling regime has to be applied. LDI generated plasmons are excluded for our experiment since this process develops on the time scale of ion acoustic waves (ca. 10 ps) and electron

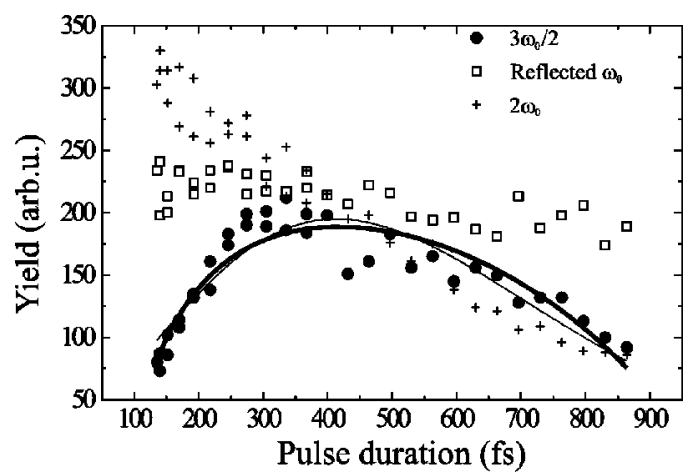

FIG. 2. Yield of $3 \omega_{0} / 2$, the $2 \omega_{0}$, and the reflected $\omega_{0}$ signal as a function of the pulse duration of the incident Ti:Sa laser for constant fluence. The thick curve represents a theoretical fit with saturation, while the thin curve is without saturation for the 3/2-harmonic signal.

plasma wave propagation is not significant (see below). Assuming a direct coupling of plasmons and laser photons, the spectral broadening is due to (1) the large bandwidth of the Ti:sapphire laser $(\sim 12 \mathrm{~nm})$, (2) the plasma electron temperature which leads to an electron density range where the $3 \omega_{0} / 2$ is generated, and (3) might be influenced by saturation which leads to an increased spectral broadening.

Figure 2 shows the measured signal of the fundamental (open squares), the second harmonic (crosses), and the 3/2harmonic radiation (filled circles) as a function of the incident Ti:Sa laser pulse duration $(\tau)$ for a constant fluence. The pulse duration was varied in the range of $135 \mathrm{fs}-900 \mathrm{fs}$ by choosing different positions of the compressor gratings. The measurement shows both directions from the optimal compressor position and therefore includes positive and negative chirp. The resulting signals were identical, consequently the generation process of the harmonics and the reflectivity do not depend on the sign of the chirp of the incoming laser pulse. Since the energy and the size of the focus were kept constant, the intensity is inversely proportional to the pulse length. The measurement shows that the $\omega_{0}$-signal remained almost constant, indicating that the reflectivity did not change in the applied intensity range. The second harmonic signal increases with shorter pulses, as found previously by others. ${ }^{15}$ By contrast, the yield of the $3 \omega_{0} / 2$ radiation has a maximum at $(350 \pm 50)$ fs.

The key point in understanding the peculiar behavior of the 3/2-yield is that due to the prepulses, the linearly polarized intense laser pulses interacted with an extended inhomogeneous plasma with long density scale-length to generate the $3 \omega_{0} / 2$. The mechanism for the $3 \omega_{0} / 2$ generation includes two steps. First, a plasmon is generated with $\omega_{0} / 2$ frequency by SRS and TPD just below the quarter-critical density. ${ }^{3}$ Second, the $3 \omega_{0} / 2$ light is produced via a coupling of the plasmons and the laser photons. The effect of plasmon propagation in the inhomogeneous plasma is generally important because in most cases the plasmons of significant growth generated by TPD do not satisfy the matching conditions (momentum and energy conservation, dispersion relations) of the 3/2-harmonic generation process. ${ }^{16}$ Direct coupling is not possible if $\beta=1.41$ $\times 10^{14} T_{e}^{2}(\mathrm{keV}) /\left[I\left(\mathrm{~W} / \mathrm{cm}^{2}\right) \lambda^{2}(\mu \mathrm{m})\right]>1$, which is typically 
the case for nanosecond laser-plasma interaction. ${ }^{17} T_{e}, I$, and $\lambda$ are the electron temperature, the laser intensity, and wavelength, respectively. $\sqrt{\beta}$ is approximately the normalized thermal velocity squared $\left[v_{e}^{2} / c_{0}^{2}=T_{e} /\left(m_{e} c_{0}^{2}\right), m_{e}\right.$ is the electron mass and $c_{0}$ is the light speed in vacuum] divided by the normalized quiver velocity $\left(v_{\mathrm{osc}} / c_{0}\right.$ $\left.=\sqrt{I \lambda^{2} /\left(1.38 \times 10^{18} \mathrm{~W} \mathrm{~cm}^{-2} \mu \mathrm{m}^{2}\right)}\right)$. In our case $\beta \approx 2$ $\times 10^{-3}-2 \times 10^{-2}$ is estimated for $\lambda=0.8 \mu \mathrm{m}, \mathrm{T}_{e}=1 \mathrm{keV}$, $\mathrm{I} \sim 10^{16}-10^{17} \mathrm{~W} / \mathrm{cm}^{2}$, which indicates that direct coupling is possible in our experiments. ${ }^{17}$ We performed a detailed $\mathrm{k}$-space analysis ${ }^{10}$ for our experimental conditions $\left(45^{\circ}\right.$ angle of incidence, $45^{\circ}$ angle of observation) which shows that plasmon propagation does not play an important role in $3 \omega_{0} / 2$ generation due to the short pulse duration and the long density scale length. In the following, plasmon propagation is neglected in the analysis of our data.

The threshold intensity for SRS is in general higher than for TPD, ${ }^{12}$ but in our case the applied intensity is high enough to invoke both processes. It is readily shown for SRS that in the vicinity of $1 / 4 n_{c}$ the momentum of the incident light wave is almost completely transferred to the plasma wave. However, these plasmons do not fulfill the matching conditions for $3 \omega_{0} / 2$. We conclude that SRS is not the dominant production process for the 3/2-harmonic radiation observed under our experimental conditions.

By contrast, TPD generates two plasmons with a frequency of about $\omega_{0} / 2$ which take up the momentum of the incident photon. TPD provides a wide range of plasmon wave vectors, which allows the generation of 3/2-harmonic radiation in a large solid angle and also in our geometry. The observed 3/2-emission is therefore assumed to be due to the coupling of plasmons of $\omega_{0} / 2$-frequency, which are generated by TPD in the vicinity of $1 / 4 n_{c}$ and incoming laser photons. The 3/2-intensity is proportional to the intensity of the laser beam at $1 / 4 n_{c}$ and to the square of the electron plasma wave amplitude.

In the linear regime, the plasma wave amplitude grows from thermal noise level $\left(n_{\mathrm{th}}\right)$ exponentially in time $n_{\mathrm{TPD}}(t)=n_{\text {th }} \exp (\gamma t)$. To calculate the instantaneous amplitude, we take the maximum TPD growth rate $\gamma$ in an inhomogeneous plasma, given in Refs. 3 and 18 extended with damping terms

$$
\gamma=\frac{k_{0} v_{\mathrm{osc}}}{4}-\sqrt{\frac{0.65 k_{0}}{L}} v_{e}-1.06 \times 10^{-2} \frac{Z \omega_{p}^{4} \ln \Lambda}{n_{e} v_{e}^{3}}-\gamma_{L},
$$

where $k_{0} \approx(\sqrt{3} / 2) \omega_{0} / c_{0}$ is the light wave vector at $1 / 4 n_{c} ; Z$ is the ionization charge state; $\omega_{p}$ is the local plasma frequency, and $\ln \Lambda \approx 7$ is the Coulomb-logarithm. $L$ $=n /|d n / d x|$ is the electron density scale length at $1 / 4 n_{c}$. The first term on the right hand side is the maximum growth rate in homogeneous plasmas $(L=\infty)$ without damping. The second term is due to a smaller interaction range in the inhomogeneous plasma, the third is the collisional damping and the fourth is the Landau damping, which is negligible if the plasmon wave vector satisfies $k<4 k_{0}$. The second and third terms introduce an intensity threshold. A preliminary estimate of the amplification using Eq. (1) assuming pure

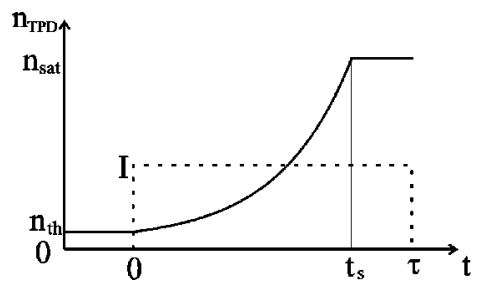

FIG. 3. The electron plasma wave amplitude ( $\left.n_{\mathrm{TPD}}\right)$ and the laser intensity $(I)$ as a function of time. The time $t_{s}$ marks the point where saturation $\left(n_{\text {sat }}\right)$ is reached from the initial thermal noise $\left(n_{\mathrm{th}}\right)$ and $\tau$ is the pulse duration.

exponential growth $\left(\lambda_{0}=790 \mathrm{~nm}, I=2 \times 10^{17} \mathrm{~W} / \mathrm{cm}^{2}, T_{e}\right.$ $=1 \mathrm{keV}, L=120 \mu \mathrm{m}, Z=4, t=135 \mathrm{fs}$ ) shows that for the experiment described here, 41 e-foldings are obtained and hence saturation has to be taken into account. In order to include saturation effects, we assume a saturation value for the plasma wave amplitude $\left(n_{\text {sat }}\right)$ which is reached at a time $t_{s}$ (Fig. 3). For times $t<t_{s}$, the instability grows exponentially; for $t \geqslant t_{s}$, the amplitude is treated as constant. The interaction time is assumed to be equal to the laser pulse duration $(\tau)$. The measured $3 \omega_{0} / 2$ pulse energy for various pulse length is given by

$$
E_{3 / 2} \sim \int_{0}^{\tau} \operatorname{In}_{\mathrm{TPD}}^{2}(t) d t
$$

with $I=I_{0} \tau_{0} / \tau$ where $I_{0}$ is the intensity at a pulse duration $\tau_{0}$. Combining Eqs. (1) and (2), we readily obtain the following expressions for $E_{3 / 2}$ :

(a) without saturation

$E_{3 / 2}(\tau)=a \frac{\exp [b \sqrt{\tau}-c \tau]}{b \sqrt{\tau}-c \tau}$

(b) with saturation

$$
E_{3 / 2}(\tau)=d\left[1+\frac{1-2 \ln \left(n_{\mathrm{sat}} / n_{\mathrm{th}}\right)}{b \sqrt{\tau}-c \tau}\right] ;
$$

where

$$
\begin{aligned}
a, b= & 6.94 \times 10^{-10} \sqrt{I_{0}\left(\mathrm{~W} / \mathrm{cm}^{2}\right) \tau_{0}(\mathrm{fs})} \mathrm{fs}^{-1 / 2}, \\
c= & \left\{4.98 \times 10^{-2} \sqrt{T_{e}(\mathrm{keV}) /\left(L_{\mathrm{eff}}(\mu \mathrm{m}) \lambda_{0}(\mu \mathrm{m})\right)}\right. \\
& \left.+1.8 \times 10^{-4} \mathrm{Z} /\left(T_{e}^{3 / 2}(\mathrm{keV}) \lambda_{0}^{2}(\mu \mathrm{m})\right)\right\} \mathrm{fs},
\end{aligned}
$$

and $d$ are fitting parameters. Henceforth, we will treat $\tau_{0}$, $Z \approx 4, L_{\text {eff }}$, and $\lambda_{0}$ as given and $I_{0}$ and $T_{e}$ as free parameters. From $b$ the intensity at $1 / 4 n_{c}\left(I_{0}\right)$ is determined for a pulse duration of $\tau_{0}$. (This may differ from the nominal focused laser intensity in vacuum due to nonlinear propagation effects.) $L_{\text {eff }}$ is an effective scale length that takes the oblique incidence of the laser beam into account. ${ }^{19}$

Interferometric measurements were performed with the second harmonic of the fs laser and a Mach-Zehnder interferometer in order to characterize the preformed plasma. The intensity of the preplasma generating laser pulse was varied from $5 \times 10^{14} \mathrm{~W} / \mathrm{cm}^{2}$ up to $10^{17} \mathrm{~W} / \mathrm{cm}^{2}$. From the measurements a density scale-length in the range of $100 \mu \mathrm{m}-200$ $\mu \mathrm{m}$ is inferred for the $12.5 \mathrm{~ns}$ time delay. 
The thin and thick curves shown in Fig. 2 are the result of respective fits using Eqs. (3) and (4) to the experimental data. A good agreement between measurement and theory is obtained in both cases. However, from these curves alone, it cannot be concluded whether saturation is reached or not. The first part in Eq. (1) is responsible for an increasing 3/2signal with the pulse duration for incident pulses shorter than $350 \mathrm{fs}$, while for longer pulses the inhomogeneous and collisional parts lead to a decreasing signal. Since it is expected that LDI plays a minor role on femtosecond timescales, linear theory should apply to even higher $e$-foldings than for long laser pulses until the instability saturates through, for example, wavebreaking. The deduced intensities using $\tau_{0}$ $=135 \mathrm{fs}$ are without saturation: $(3.9 \pm 0.5) \times 10^{15} \mathrm{~W} / \mathrm{cm}^{2}$ with saturation assuming $10 e$-foldings saturation level [(2 $\left.\ln \left(n_{\text {sat }} / n_{\text {th }}\right)=10\right]: \quad(2.4 \pm 0.1) \times 10^{16} \mathrm{~W} / \mathrm{cm}^{2} \quad$ and 20 $e$-foldings: $(1.05 \pm 0.04) \times 10^{17} \mathrm{~W} / \mathrm{cm}^{2}$. There are more definite but much more complicated theories of TPD ${ }^{17,19}$ with slightly different maximum growth rates which essentially show the same behavior as the model of Ref. 18 and the experimental data.

We calculated the collisional absorption of the incident laser pulse for propagation up to the $1 / 4 n_{c}$ and found that it is negligible, but ionization defocusing in the preplasma is significant for the fundamental. Refraction of the threehalves harmonic in $45^{\circ}$ by the laser generated density profile is negligible due to the perpendicular propagation to the laser direction. Estimates show that the intensity at $1 / 4 n_{c}$ is reduced by about one to two orders of magnitude due to ionization defocusing and therefore an intensity of $10^{15}-10^{16} \mathrm{~W} / \mathrm{cm}^{2}$ is expected inside the plasma in agreement with our measurement. The spectral shift of the $3 \omega_{0} / 2$ radiation may be attributed to the ionization blue shift of the fundamental. The electron temperature obtained from the fit parameter $c$ using Eq. (3) is on the order of $\sim 230 \mathrm{eV}$. It should be stressed that even with saturation the duration of the exponential growth is in our experiment at least $80 \%$ of the laser pulse length (see Fig. 3), in contrast to ns-laser experiments where it is approximately $1 \% .^{12}$ Since most of the interaction time is within an exponential growth the fits with and without saturation are similar.

In summary, we have performed the first experimental investigation of intense femtosecond laser generated $3 \omega_{0} / 2$ radiation in dense long scale length plasmas. The dominant production process for the 3/2-harmonic radiation is the two plasmon decay. The good agreement of the theoretical models with the experimental data-in particular the occurrence of a maximum in the 3/2-yield as a function of $\tau$ (Fig. 2) provides a clear experimental verification of the TPD growth rate in an inhomogeneous plasma in the linear regime-Eq. (1).

\section{ACKNOWLEDGMENTS}

We thank K.-U. Amthor for helping us in the interferometric density measurements. M.S.J. acknowledges the support by the German Service for Academic Exchange (DAAD).

This work was supported by the Deutsche Forschungsgemeinschaft (TH 700/1-2).

${ }^{1}$ P. Gibbon and E. Förster, Plasma Phys. Controlled Fusion 38, 769 (1996). ${ }^{2}$ W. Theobald, R. Hässner, C. Wülker, and R. Sauerbrey, Phys. Rev. Lett. 77, 298 (1996).

${ }^{3}$ W. L. Kruer, The Physics of Laser Plasma Interactions (Addison-Wesley, New York, 1988).

${ }^{4}$ U. Teubner, J. Bergmann, B. van Wonterghem, F. P. Schäfer, and R. Sauerbrey, Phys. Rev. Lett. 70, 794 (1993).

${ }^{5}$ J. D. Kmetec, C. L. Gordon III, J. J. Macklin, B. E. Lemoff, G. S. Brown, and S. E. Harris, Phys. Rev. Lett. 68, 1527 (1992).

${ }^{6}$ N. A. Ebrahim, H. A. Baldis, C. Joshi, and R. Benesch, Phys. Rev. Lett. 45, 1179 (1980).

${ }^{7}$ D. W. Phillion, E. M. Campbell, K. G. Estabrook, G. E. Phillips, and F. Ze, Phys. Rev. Lett. 49, 1405 (1982).

${ }^{8}$ W. Seka, B. B. Afeyan, R. Boni, L. M. Goldman, R. W. Short, K. Tanaka, and T. W. Johnston, Phys. Fluids 28, 2570 (1985).

${ }^{9}$ P. E. Young, B. F. Lasinski, W. L. Kruer, E. A. Williams, K. G. Estabrook, E. M. Campbell, R. P. Drake, and H. A. Baldis, Phys. Rev. Lett. 61, 2766 (1988).

${ }^{10}$ J. Meyer and Y. Zhu, Phys. Rev. Lett. 71, 2915 (1993).

${ }^{11}$ D. A. Russell and D. F. DuBois, Phys. Rev. Lett. 86, 428 (2001).

${ }^{12}$ H. A. Baldis and C. J. Walsh, Phys. Fluids 26, 1364 (1983).

${ }^{13}$ C. B. Darrow, C. Coverdale, M. D. Perry, W. B. Mori, C. Clayton, K. Marsh, and C. Joshi, Phys. Rev. Lett. 69, 442 (1992); C. Rousseaux, G. Malka, J. L. Miquel, F. Amiranoff, S. D. Baton, and Ph. Mounaix, ibid. 74, 4655 (1995).

${ }^{14}$ E. E. Fill, J. Opt. Soc. Am. B 11, 2241 (1994).

${ }^{15}$ I. B. Földes, J. S. Bakos, G. Veres, Z. Bakonyi, T. Nagy, and S. Szatmári, IEEE J. Sel. Top. Quantum Electron. 2, 776 (1996).

${ }^{16}$ J. Meyer, Phys. Fluids B 4, 2934 (1992).

${ }^{17}$ A. Simon, R. W. Short, E. A. Williams, and T. Dewandre, Phys. Fluids 26, 3107 (1983).

${ }^{18}$ H. A. Baldis, E. M. Campbell, and W. L. Kruer, in Handbook of Plasma Physics, edited by N. M. Rosenbluth and R. G. Sagdeev (Elsevier Science, New York, 1991), Vol. 3, pp. 397-408.

${ }^{19}$ B. B. Afeyan and E. A. Williams, Phys. Plasmas 4, 3827 (1997). 